\title{
Clintonite-bearing assemblages in chondrodite marbles from the contact aureole of the Třebíč Pluton, Moldanubian Zone, Bohemian Massif
}

\author{
Asociace obsahující clintonit v chondroditových mramorech moldanubika \\ z kontaktní aureoly třebíčského plutonu, Český masiv
}

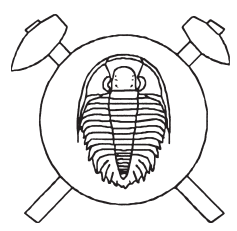

(6 figs, 4 tabs)

STANISLAV HOUZAR ${ }^{1}$ - MILAN NOVÁK ${ }^{2}$

${ }^{1}$ Department of Mineralogy and Petrography, Moravian Museum, Zelný trh 6, CZ-659 37 Brno, Czech Republic; shouzar@mzm.cz ${ }^{2}$ Institute of Geological Sciences, Masaryk University, Kotlářská 2, CZ-611 37 Brno, Czech Republic; mnovak@sci.muni.cz

\begin{abstract}
Clintonite is a minor to accessory mineral in chondrodite marbles. They represent a rare type of metacarbonate rocks in the Varied Unit of the Moldanubian Zone, forming thin bodies enclosed in migmatites. Clintonite occurs exclusively in marbles from contact aureole of melanocratic ultrapotassic granites (durbachites) of the Třebíč Pluton. Chondrodite marbles consist of dominant calcite, less abundant dolomite; amounts of silicates vary from $\sim 5$ to 30 vol. \%. The early mineral assemblage Dol $+\mathrm{Cal}+\mathrm{Prg} \pm \mathrm{Phl}$ is replaced by the assemblage $\mathrm{Chn}+\mathrm{Cli}+\mathrm{Cal} \pm \mathrm{Chl} \mathrm{I} \pm \mathrm{Spl}$. Accessory minerals include fluorapatite, diopside, tremolite, pyrrhotite, and rare zircon and baddeleyite. Violet fluorite occurs on late fissures. Clintonite forms colourless to pale green flakes and sheaf-like aggregates, up to $2 \mathrm{~mm}$ in size. It has extraordinary high Si (2.739-2.986 apfu) and Si/Al ratio (0.52-0.60). The contents of $\mathrm{Fe}_{\text {tot }}(0.041-0.128 \mathrm{apfu}), \mathrm{Na}(0.035-0.134 \mathrm{apfu})$, Ti (0.004-0.024 apfu) and K ( $\leq 0.005 \mathrm{apfu})$ are low. High concentrations of F $(0.437-1.022 \mathrm{apfu})$ corresponding up to $26 \%$ of the F-component are the highest ever-recorded in clintonite. Mineral reactions producing clintonite, which is closely associated with more abundant chondrodite $\left(\mathrm{X}_{\mathrm{F}}=0.45-0.67\right)$, are not clear in detail, because textural relations often do not show any apparent replacement features. The simplified reactions involving diopside, pargasite and/or phlogopite: (5) $\mathrm{Dol}+\mathrm{Phl}+\mathrm{Di}+\mathrm{H}_{2} \mathrm{O}=\mathrm{Cli}+\mathrm{Chn}+\mathrm{Chl} \mathrm{I}+\mathrm{Cal}+\mathrm{CO}$, $+\mathrm{K}_{2} \mathrm{O},(6,7) \mathrm{Dol}+\mathrm{Prg}(\mathrm{and} / \mathrm{or} \mathrm{Phl})=\mathrm{Cli}+\mathrm{Chn}+\mathrm{Cal}+\mathrm{CO}_{2}+\mathrm{K}_{2} \mathrm{O} \pm \mathrm{Na}_{2} \mathrm{O}$, and (8) Dol $+\mathrm{Phl}(\operatorname{and} / \mathrm{or} \mathrm{Prg})+\mathrm{SiO} 2 \mathrm{~F}=\mathrm{Cli}+\mathrm{Chn}+\mathrm{Spl}$ $+\mathrm{Cal}+\mathrm{CO}_{2}+\mathrm{K}_{2} \mathrm{O} \pm \mathrm{Na}_{2} \mathrm{O}$ seem the most probable. Retrograde chlorite II formed by the reaction: (9) $\mathrm{Chn}+\mathrm{Cli}+\mathrm{CO}_{2}=\mathrm{Chl}+\mathrm{Cal}$. $\mathrm{Mineral}$ assemblages of clintonite-bearing chondrodite marbles are product of regional-scale contact ("periplutonic") LP metamorphism at: $\mathrm{P} \sim 2-4$ kbar; $\mathrm{T} \sim 620-730{ }^{\circ} \mathrm{C}$. The reactions might be buffered internally, if pargasite and/or phlogopite were a source of $\mathrm{F}$ and $\mathrm{Al}$; however, high modal content of chondrodite in most samples is likely related to influx of $\mathrm{H}_{2} \mathrm{O}, \mathrm{Si}$ and $\mathrm{F}$ from an external source. It is also supported by isotopic composition of calcite $\left(\delta^{13} \mathrm{C}_{\text {calcite }}=-0.6--4.2 \% \mathrm{PDB} ; \delta^{18} \mathrm{O}_{\text {calcite }}=12.5-15.7 \% \mathrm{SMOW}\right)$. Fluorine-rich fluids very likely stabilized the assemblage chondrodite + clintonite relative to compositionally equivalent assemblages involving Mg-silicate (forsterite and/or clinohumite) + chlorite (and/or spinel) + calcite.
\end{abstract}

Key words: clintonite; chemical composition; fluorine; chondrodite marble; Moldanubian Zone; Bohemian Massif

\section{Introduction}

Clintonite is a rare trioctahedral brittle mica with the ideal composition $\mathrm{Ca}\left(\mathrm{Mg}_{2} \mathrm{Al}\right)_{3}\left(\mathrm{SiAl}_{3}\right) \mathrm{O}_{10}(\mathrm{OH})_{2}$. It occurs chiefly in contact metamorphosed dolomite marbles, calcic and magnesian skarns (e.g. Burnham 1959, Shabynin 1973, Bucher-Nurminen 1976, Rice 1979, Bucher - Frey 1994, Sugaki et al. 2000, Woodford et al. 2001) and rarely in rodingites (Rice 1983, Dubińska 1997, Dubińska et al. 2004). Ackermand et al. (1986) described for the first time clintonite from regional metamorphic rock with the assemblage $\mathrm{Cal}+\mathrm{Ol}+\mathrm{Cpx}+\mathrm{Spl}+\mathrm{Sph}+\mathrm{Chl}$. More recently, Kato et al. (1997) described clintonite in equilibrium with primary garnet and from symplectites between garnet and clinohumite in eclogite included in marble of the UHP Su-Lu terrane, China.

Phase relations and mineral reactions of clintonite were only sporadically studied experimentally (e.g., Olesch Seifert 1976, Zharikov - Khodorevskaya 1987). It exhibits a wide stability field in PTX diagrams but restricted to low $\mathrm{X}_{\mathrm{CO} 2}$. Clintonite also requires specific chemical composition characterized by very high $\mathrm{Al} / \mathrm{Si}$ ratio and low activity of $\mathrm{Na}$ and K (Olesch - Seifert 1976, Rice 1983, Ulmer 1983, Ackermand et al. 1986, Alietti et al.
1997, Mottana et al. 2002). Its crystal chemistry was studied in detail by MacKinney et al. (1988), but the authors did not provide any information about mineral assemblages of the studied clintonite samples. Only a low number of chemical analyses of clintonite have been published up to now and the data concerning concentrations of $\mathrm{F}$ and valence of $\mathrm{Fe}$ are rather exceptional (Rice 1979, MacKinney et al. 1988, Alietti et al. 1997, Grew et al. 1999). Based on the mineral assemblages and textural relations, mineral reactions producing clintonite involve calcite, spinel, forsterite and diopside (Rice 1979), less commonly also phlogopite, pargasite, chlorite and monticellite (Bucher-Nurminen 1976, U1mer 1983).

Novák - Houzar (1996) published the first comprehensive description of clintonite closely associated with chondrodite from marbles at Tasov (Fig. 1), and two new occurrences were found later in similar marbles at Sokolí and Č́halín. All these localities of clintonite are situated in migmatites within contact aureole of the Třebíc Pluton (Houzar - Novák 2006). Here we present data concerning mineral assemblages, crystal chemistry of clintonite and associated minerals, and geological position of clintonite-bearing chondrodite marbles. 


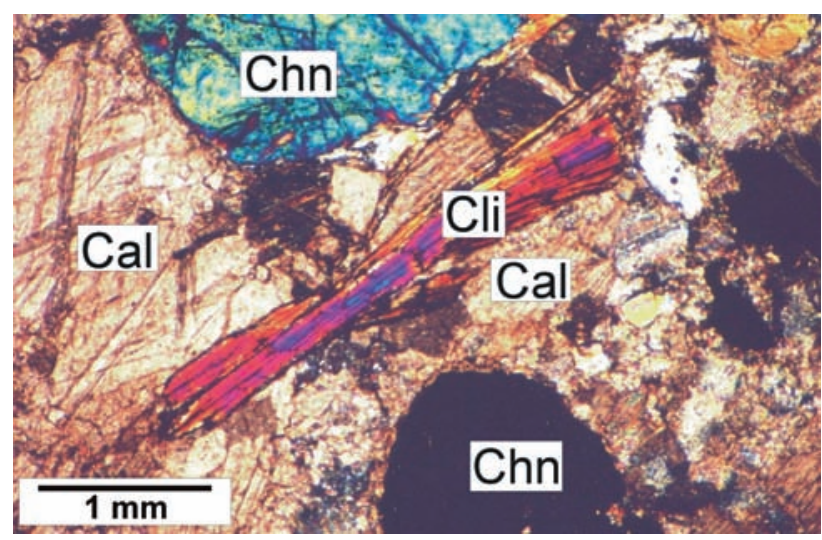

Fig. 1 Sheaf-shaped aggregates of clintonite enclosed in calcite, with isometric grains of chondrodite. Tasov near Třebíč, crossed polarizers.

\section{Geologic setting}

The examined chondrodite marbles occur in the Varied Unit of the Moldanubian Zone (Fig. 2). This unit is built dominantly by metapelites with the mineral assemblages $\mathrm{Bt}+\mathrm{Qtz}+\mathrm{Pl}+\mathrm{Kfs} \pm \mathrm{Sil} \pm \mathrm{Grt} \pm \mathrm{Crd}$, which are migmatized to a variable degree, locally with dominance of metatect over melanosome characterized by the assemblage $\mathrm{Kfs}+\mathrm{Qtz}+\mathrm{Pl}+\mathrm{Bt} \pm \mathrm{Grt} \pm \mathrm{Crd}$. Metapelites contain common intercalations of various rocks: calcite and dolomite marbles, locally diopside gneisses ( $\mathrm{Di}+\mathrm{Qtz}+\mathrm{Pl}+\mathrm{Cal}+\mathrm{Ttn} \pm \mathrm{Phl}$ $\pm \mathrm{Amf}$ ), a variety of calc-silicate rocks $(\mathrm{Di}+\mathrm{Pl}+\mathrm{Kfs}+\mathrm{Ttn}$ $\pm \mathrm{Wo} \pm \mathrm{Grs} \pm \mathrm{Cal}$ ), graphite quartzites (Qtz $+\mathrm{Gr}+\mathrm{Kfs}+\mathrm{Sil}+\mathrm{Ms}$ $\pm \mathrm{Bt} \pm \mathrm{Pl})$ and amphibolites (Amp+Pl+Di+Ttn).

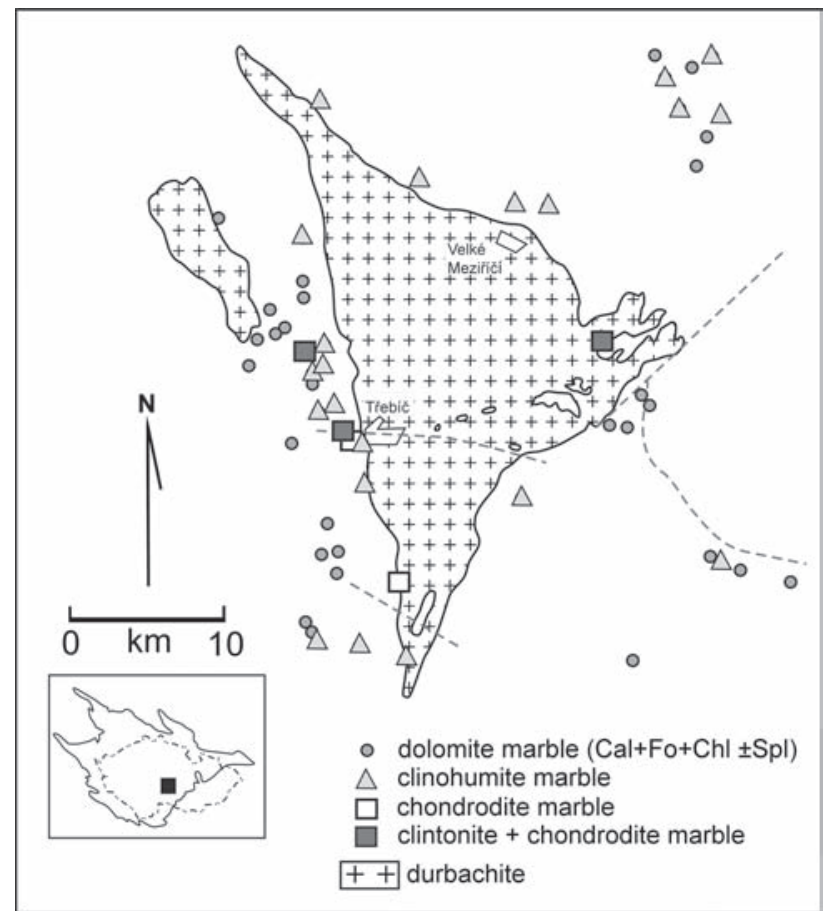

Fig. 2 Geological situation of dolomite marbles in the Třebíč Pluton area.
Several distinct types of marbles were distinguished in the eastern part of the Moldanubicum within the area of the Třebíč Pluton (Houzar 2004, Novák 1989). Abundant Si-poor dolomite marbles (15-22 wt.\% MgO) with the mineral assemblage $\mathrm{Dol}+\mathrm{Cal}+\mathrm{Phl}+\mathrm{Tr}+\mathrm{Fo}+\mathrm{Chu}+\mathrm{Spl}+\mathrm{Chl}$, less abundant calcite marbles $(<3$ wt. $\% \mathrm{MgO})$ with the mineral assemblage $\mathrm{Cal}+\mathrm{Phl}+\mathrm{Tr}+\mathrm{Di}+\mathrm{Qtz}+\mathrm{Kfs}+\mathrm{Wo}+\mathrm{Ttn}$, and rare chondrodite marbles $(<8 \mathrm{wt} . \% \mathrm{MgO})$ with the assemblage $\mathrm{Cal}+\mathrm{Dol}+\mathrm{Phl}+\mathrm{Prg}+\mathrm{Chn}+\mathrm{Di}+\mathrm{Spl}+\mathrm{Chl}$ are documented.

Three distinct metamorphic events were recognized in the dolomite marbles, the only metacarbonate rocks studied in detail in this region. (i) The early event is represented by the assemblages: $\mathrm{Cal}+\mathrm{Dol}+\mathrm{Phl}+\mathrm{Tr} \pm \operatorname{Prg} \pm \mathrm{Di}$ developed in almost all marbles; these minerals seem to be locally in equilibrium. They may have formed at about $\mathrm{T}_{\text {min. }}=660{ }^{\circ} \mathrm{C}$ for $\mathrm{P}=600 \mathrm{MPa}$ (Novák 1989, Houzar 2004). (ii) The dominant metamorphic event is represented by the assemblages: $\mathrm{Cal}+\mathrm{Dol}+\mathrm{Fo}+\mathrm{Chl} \mathrm{I} \pm \mathrm{Spl} \pm \mathrm{Chu}$, with accessory $\mathrm{Mg}$-ilmenite, geikielite and rare baddeleyite, occurring almost exclusively in dolomitic marbles. This regional-scale contact LP metamorphism (Kerrick 1991) exhibits well-developed zoning spatially related to the Třebíč Pluton (Novák - Houzar 1996). The localities of clintonite examined are located within the clinohumite zone characterized by the equilibrium mineral assemblages: $\mathrm{Dol}+\mathrm{Cal}+\mathrm{Chu}+\mathrm{Spl} \pm \mathrm{Fo} \pm \mathrm{Chl}$ I. These assemblages are locally univariant and exceptionally invariant (Novák - Houzar 1996, Houzar 2004). The PTX conditions of this event were estimated at $\mathrm{T}=540-620^{\circ} \mathrm{C}$ for $\mathrm{P}_{\text {fluid }} 200$ $\mathrm{MPa}, \mathrm{X}_{\mathrm{CO} 2}=0.1-0.4$; Novák - Houzar 1996), or $\mathrm{T}_{\max .}=$ $660-750^{\circ}$ for $\mathrm{P}_{\text {fluid }}=300-400 \mathrm{MPa}$ (Houzar 2004). Chlorite II was formed in a final stage of this event. (iii) Retrograde stage of metamorphism is represented by formation of abundant antigorite instead of forsterite at $\mathrm{T}<$ $\sim 450{ }^{\circ} \mathrm{C}$ and very low $\mathrm{X}_{\mathrm{CO} 2}<0.05$ (Houzar - Novák 2001).

The Třebíč Pluton occurring in close vicinity of all clintonite localities belongs to the ultrapotassic plutonic rocks $\left(\mathrm{MgO}>3\right.$ wt. $\%, \mathrm{~K}_{2} \mathrm{O} / \mathrm{Na}_{2} \mathrm{O}>2$; Foley et al. 1987) of the durbachite series. The bulk composition of the Třebíč Pluton is characterized by a metaluminous signature $(\mathrm{ASI}=0.85-0.93)$, high contents of $\mathrm{K}_{2} \mathrm{O}$ (5.2-6.5 wt.\%), $\mathrm{MgO}$ (3.3-10.4 wt.\%), $\mathrm{P}_{2} \mathrm{O}_{5}(0.47-0.98$ wt.\%), Rb (330$410 \mathrm{ppm}), \mathrm{Ba}(1100-2470 \mathrm{ppm}), \mathrm{U}$ (6.7-26.2 ppm), Th (28.2-47.7 ppm), and Cr (270-650 ppm) (Holub 1997, Holub et al. 1997). The radiometric dating indicates its Lower Carboniferous age of $343 \pm 6 \mathrm{Ma}(\mathrm{Pb}-\mathrm{Pb}$ zircon; Holub et al. 1997), 338 \pm 4 Ma (U-Pb zircon; Kotková et al. 2003).

\section{Methods}

Chemical composition of minerals was studied on electron microprobe Cameca SX-100 (GÚDŠ, Bratislava; operator V. Kollárová). The following analytical conditions were applied: accelerating voltage $15 \mathrm{kV}$, beam diameter 5-10 $\mu \mathrm{m}$, beam current $20 \mathrm{nA}$ and counting times 
20 s. The following standards and $\mathrm{K} \alpha$ lines were used: wollastonite $(\mathrm{Si}, \mathrm{Ca}), \mathrm{TiO}_{2}(\mathrm{Ti})$, orthoclase $(\mathrm{K})$, albite $(\mathrm{Na}), \mathrm{Al}_{2} \mathrm{O}_{3}(\mathrm{Al}), \mathrm{MgO}(\mathrm{Mg})$, hematite $(\mathrm{Fe})$, rhodonite $(\mathrm{Mn}), \mathrm{BaF}_{2}(\mathrm{~F})$, barite $(\mathrm{Ba})$, and chromite $(\mathrm{Cr})$. The concentrations of $\mathrm{F}$ in amphiboles, micas and chondrodite were determined using a routine electron microprobe procedure, no special methods were employed. Hence, the concentrations obtained may show quite a high error of $\sim 10$ rel.\%. (see Ottolini et al. 2000). High totals of cations in chondrodite may suggest presence of intimate intergrowths of humite and/or non-stoichiometric members of humite group (Ehlers - Hoinkes 1987, Satish-Kumar - Niimi 1998). Hence, for their identification the $\mathrm{M}_{\mathrm{Ti}} / \mathrm{Si}$ $\left(\mathrm{M}_{\mathrm{Ti}}=\mathrm{Mg}+\mathrm{Fe}^{2+}{ }_{\text {tot }}+\mathrm{Mn}+\mathrm{Ti}+\mathrm{Ca}\right)$ values given by Gaspar (1992) were used. Concentrations of $\mathrm{F}$ and $\mathrm{Mg}$ in minerals are presented as the molar ratios $\mathrm{X}_{\mathrm{F}}=\mathrm{F} /(\mathrm{F}+\mathrm{OH})$ and $\mathrm{X}_{\mathrm{Mg}}=\mathrm{Mg} /\left(\mathrm{Mg}+\mathrm{Fe}^{2+}{ }_{\text {tot }}+\mathrm{Mn}\right)$. Except clintonite (Cli) all abbreviations are according to Kretz (1983).

\section{Description of localities and textural relations of the studied samples}

Č́halín

Sample CHL-1 comes from marble boulders occurring on a field $\sim 1 \mathrm{~km} \mathrm{SW}$ of Cíhalín. Chondrodite marble forms probably thin layer(s) in cordierite-biotite gneiss with common amphibolite. Marble is white, medium grained and strongly tectonically deformed with chrysotile on fractures. It is poor in silicates $(<\sim 5$ vol. $\%$ of non-carbonate fraction). Yellow grains of chondrodite, rare colourless diopside, phlogopite partly replaced by chlorite I and pargasite occur in the marble. Scarce flakes of clintonite, up to $1 \mathrm{~mm}$ in diameter, locally replaced by late chlorite II, are intergrown with chondrodite (Fig. 3a). Rare accessory fluorapatite and zircon were found.

\section{Sokolí}

Layer of chondrodite marble (samples SOKP-I, SOKP-II), about $30-60 \mathrm{~cm}$ thick, is enclosed in metatect-rich migmatite located $\sim 1 \mathrm{~km}$ SE of the village. Medium-grained, silicate-rich grey marble with graphite contains sporadically thin veins consisting of green diopside and minor quartz. Close to the contact with the migmatite, graphite-free marble is white and contains low amount of silicates. Chondrodite forms light yellow anhedral grains, 1-5 $\mathrm{mm}$ in diameter, and rarely up to $1 \mathrm{~cm}$ large, enclosed in calcite. It shows locally polysynthetic twinning. Flakes of clintonite, $0.3-1 \mathrm{~mm}$ in diameter, are closely associated with chondrodite, and commonly replaced by late colourless chlorite II (Fig. 3b). It locally forms complete pseudomorphs after clintonite. Rather common sheets of colourless, yellowish-brown and greenish phlogopite, up to $5 \mathrm{~mm}$ in diameter, are developed on foliation planes of marble. It also occurs as small inclusions in calcite, chondrodite and chlorite. Pargasite forms small colour-
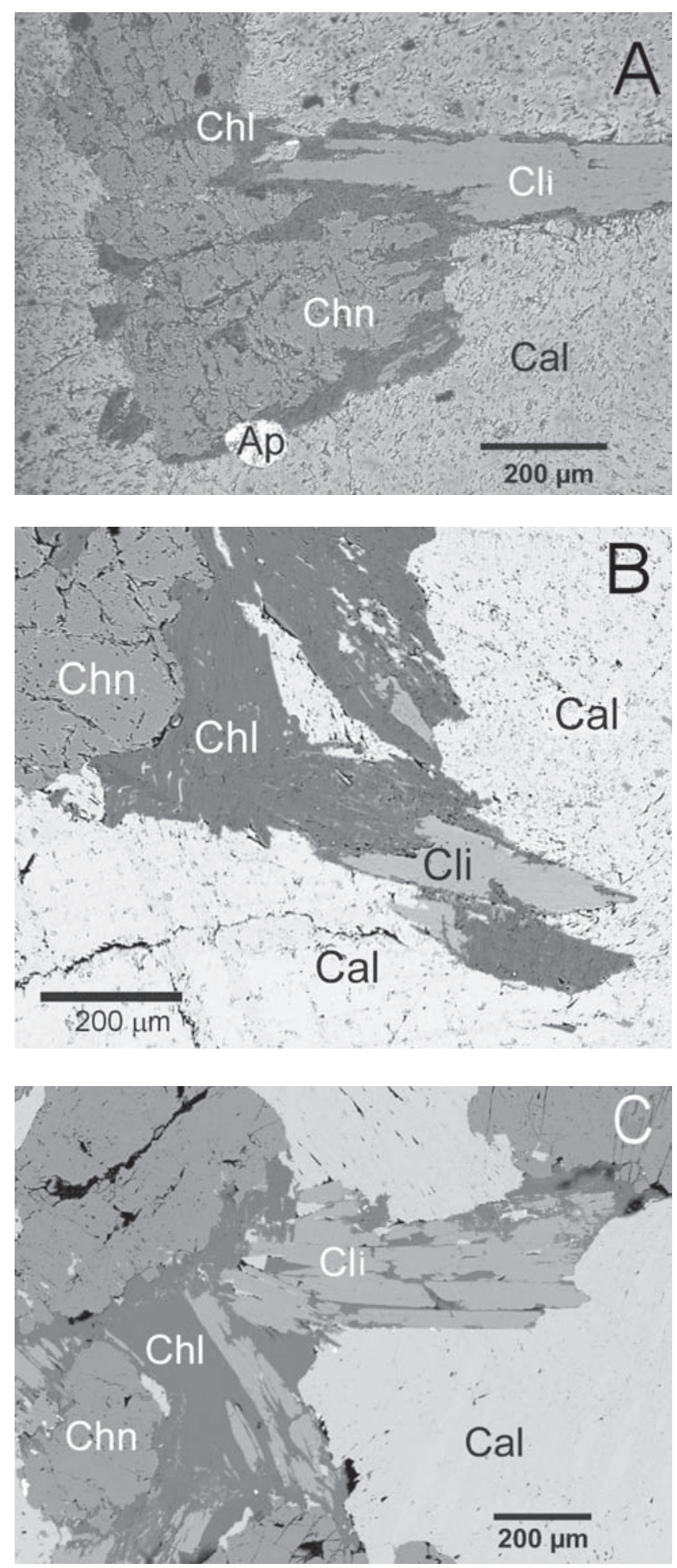

Fig. 3 BSE images of the assemblage clintonite+chondrodite+ +calcite+chlorite II: A) Č́ihalín, B) Sokolí, C) Tasov.

less long prismatic crystals locally overgrown by chondrodite, or minute greenish-grey lenticular aggregates, up to $3 \mathrm{~cm}$ in size. Colourless diopside occurs as sporadic isolated grains and small veins. Euhedral octahedrons of violet spinel, $\leq 0.2 \mathrm{~mm}$ in size, microscopic accumulations of fluorapatite and scarce late violet fluorite were found in the marble. 
Tasov

Chondrodite marbles (samples TAS-I, TAS-II) were found in the borehole no. 179 (Uranový průzkum Company) in the depth $\sim 500 \mathrm{~m}$. Marbles are hosted in migmatized garnet-biotite gneisses with rare small bodies of amphibolite and serpentinite. This rock complex is located below tabular body of durbachite of the Třebíč Pluton. Chondrodite marbles most probably form a small portions or bands rich in silicates $(>\sim 30$ vol. $\%$ of non-carbonate fraction) in large calcite-dolomite marble body, $\sim 20 \mathrm{~m}$ thick. Clintonite forms colourless and greenish sheaf-shaped aggregates, about $0.5-3 \mathrm{~mm}$ long, enclosed in or closely associated with chondrodite (Fig. 3c). It is replaced by chlorite II along contact with chondrodite, whereas clintonite enclosed solely in calcite is always fresh. Chondrodite commonly overgrows phlogopite, chlorite I and pargasite, which are locally found as tiny inclusions. It also forms lobate grains, veinlets on intergranulars connecting in minute aggregates. Accessory green spinel forms euhedral octahedral crystals, $\leq 0.4 \mathrm{~mm}$ in size. Chondrodite-bearing white marbles evidently replace darker marbles with common aggregates of long-prismatic greyish-green pargasite (or edenite) accompanied by less abundant phlogopite. Thin coatings of violet fluorite+calcite on marble fractures represent the latest mineralization.

The studied calcite-dominant chondrodite marbles contain low to moderate amount of dolomite $(<8$ wt. \% MgO). Their foliation is commonly poorly observable. The amount of non-carbonate fraction varies between $<\sim 5 \%$ (sample CHL-1) to $~ 30 \%$ (sample TAS-1). The complete mineral assemblages of clintonite commonly comprise calcite, dolomite, phlogopite, pargasite, rarely chlorite I, diopside, and several accessory minerals (Table 1). However, only calcite, chondrodite, chlorite I, and spinel were found in direct contact with clintonite. The isotopic composition of calcite from the assemblage $\mathrm{Cal}+\mathrm{Chn}+\mathrm{Cli}$ shows, compared to average sedimentary carbonates, rather lower values: in Sokolí $\delta^{13} \mathrm{C}=-0.6$ to $-0.5 \%$ (PDB), $\delta^{18} \mathrm{O}=17.3$ to $15.7 \%$ (SMOW); in Tasov $\delta^{13} \mathrm{C}=-4.2 \%$ (PDB), $\delta^{18} \mathrm{O}=12.5 \%$ (SMOW). Calcite-dolomite thermometer data (Powel et al. 1984 ) indicate that the mineral assemblages involving clintonite originated at $\mathrm{T}_{\text {max. }}=620-730{ }^{\circ} \mathrm{C}($ Houzar 2004).

\section{Chemistry of minerals from clintonite-bearing chondrodite marbles}

\section{Clintonite}

Chemical composition of clintonite with $\mathrm{X}_{\mathrm{Mg}}=0.97-0.99$ exhibits very high contents of Si (2.739-2.986 apfu), which is typical for clintonite from marbles, $\mathrm{Al}^{\mathrm{IV}}$ is rather low (5.014-5.261 apfu) as a result of the substitution
Table 1 Minerals in the individual samples of chondrodite marbles containing clintonite.

\begin{tabular}{|c|c|c|c|c|c|c|}
\hline $\begin{array}{l}\text { Locality } \\
\text { sample }\end{array}$ & & $\begin{array}{l}\text { Číhalín } \\
\text { CHL-1 }\end{array}$ & $\begin{array}{c}\text { Sokolí } \\
\text { SOKP-1 }\end{array}$ & $\begin{array}{c}\text { Sokolí } \\
\text { SOKP-2 }\end{array}$ & $\begin{array}{c}\text { Tasov } \\
\text { TAS-1 }\end{array}$ & $\begin{array}{l}\text { Tasov } \\
\text { TAS-2 }\end{array}$ \\
\hline Dolomite & Dol & $\mathrm{x}$ & $\mathrm{x}$ & $X$ & $\mathrm{x}$ & $\mathrm{X}$ \\
\hline Calcite & Cal & $\mathrm{X}$ & $\mathrm{X}$ & $\mathrm{X}$ & $\mathrm{X}$ & $\mathrm{X}$ \\
\hline Phlogopite & Phl & $\mathrm{t}$ & $\mathrm{X}$ & $X$ & $\mathrm{t}$ & $\mathrm{x}$ \\
\hline Pargasite & Prg & $\mathrm{t}$ & $\mathrm{x}$ & $\mathrm{x}$ & $\mathrm{x}$ & $\mathrm{X}$ \\
\hline Edenite & Ed & - & - & - & - & $\mathrm{x}$ \\
\hline Chlorite I & Chl & $\mathrm{X}$ & $\mathrm{x}$ & - & - & - \\
\hline Clintonite & Cli & $\mathrm{t}$ & $\mathrm{x}$ & $\mathrm{x}$ & $\mathrm{x}$ & $\mathrm{t}$ \\
\hline Chondrodite & Chn & $X$ & $\mathrm{X}$ & $X$ & X & $X$ \\
\hline Tremolite & $\mathbf{T r}$ & - & $\mathrm{t}$ & - & - & $\mathrm{t}$ \\
\hline Diopside & Di & $\mathrm{x}$ & $\mathrm{t}$ & $\mathrm{x}$ & - & - \\
\hline Spinel & Spl & - & $\mathrm{t}$ & $\mathrm{x}$ & $\mathrm{X}$ & $\mathrm{x}$ \\
\hline Graphite & Gr & - & - & $\mathrm{t}$ & - & - \\
\hline Fluorapatite & Ap & $\mathrm{t}$ & $\mathrm{x}$ & $\mathrm{t}$ & $\mathrm{t}$ & $\mathrm{t}$ \\
\hline Fluorite & Fl & - & $\mathrm{t}$ & - & $\mathrm{t}$ & $\mathrm{t}$ \\
\hline Zircon & Zrn & $\mathrm{t}$ & - & - & - & - \\
\hline Baddeleyite & Bad & - & - & - & $\mathrm{t}$ & - \\
\hline Sphalerite & Sp & - & - & - & $\mathrm{t}$ & - \\
\hline Arsenopyrite & Apy & - & - & - & - & $\mathrm{t}$ \\
\hline Pyrhotite & Po & - & - & - & $\mathrm{t}$ & $\mathrm{x}$ \\
\hline Scheelite & Sch & - & - & $\mathrm{t}$ & - & - \\
\hline Chlorite II & Chl II & $\mathrm{X}$ & $\mathrm{X}$ & $\mathrm{X}$ & $\mathrm{x}$ & $\mathrm{x}$ \\
\hline Serpentine & Srp & $X$ & - & - & - & - \\
\hline
\end{tabular}

$\mathbf{X}=$ major constituent, $\mathbf{x}=$ minor constituent, $\mathbf{t}=$ trace Abbreviations from Kretz (1983) except Cli for clintonite and Sch for scheelite

${ }^{\mathrm{IV}} \mathrm{Al}_{-1}{ }^{\mathrm{VI}} \mathrm{Mg}_{-2}{ }^{\mathrm{IV}} \mathrm{Si}^{\mathrm{VI}} \mathrm{Al}^{\mathrm{VI}} \square$ (Alietti at al. 1997, Mottana et al. 2002). The ratio $\mathrm{Si} / \mathrm{Al}^{\mathrm{IV}}(0.52$ to 0.60$)$ is much higher than the ideal value $\left(\mathrm{Si} / \mathrm{Al}^{\mathrm{IV}}=0.33\right)$. The contents of $\mathrm{Fe}^{2+}$ tot $(0.041-0.128 \mathrm{apfu})$ and $\mathrm{Na}(0.035-0.134 \mathrm{apfu})$ are low and variable, the highest values were found in Tasov (Table 2). Concentration of Ti is very low (0.004$0.024 \mathrm{apfu})$ as well as $\mathrm{K}(\leq 0.005 \mathrm{apfu})$ and $\mathrm{Cr}(0.001-$ $0.012 \mathrm{apfu}$ ), the contents of $\mathrm{Mn}$ and $\mathrm{Ba}$ were below detection limit. Clintonite exhibits high contents of $\mathrm{F}$ (0.437-1.022 apfu, $\left.X_{\mathrm{F}}=0.11-0.26\right)$, which represent the highest known values ever found (Fig. 4). High contents of $\mathrm{F}$ and high $\mathrm{Si} / \mathrm{Al}$ ratios are similar to those in clintonite from fluorine-rich marbles at the Orange County localities, New York, USA (MacKinney et al. 1988).

\section{Chondrodite}

Chondrodite exhibits high $\mathrm{X}_{\mathrm{Mg}}=0.95-0.99$, Fe varies from 0.077 apfu to 0.224 apfu; the highest contents of Fe are from chondrodite at Tasov. It is poor in $\mathrm{Ti}(0.012-$ $0.043 \mathrm{apfu}), \mathrm{Mn}(\leq 0.008 \mathrm{apfu})$ and $\mathrm{Ca}(\leq 0.004 \mathrm{apfu})$. Variable but moderate amounts of $\mathrm{F}\left(\mathrm{X}_{\mathrm{F}}=0.45-0.67\right)$ were found. Histogram representing the range of $\mathrm{M}_{\mathrm{Ti}} / \mathrm{Si}$ values shows bimodal distribution (Fig. 5). This suggests 
Fig. 4 Diagram showing the fluorine concentrations in clintonites: shaded - studied localities; dotted - clintonite from marbles in the Orange County, USA; white - other clintonites (data source: Rice 1979, MacKinney et al. 1988, Allieti et al. 1997, Grew et al. 1999, this study).

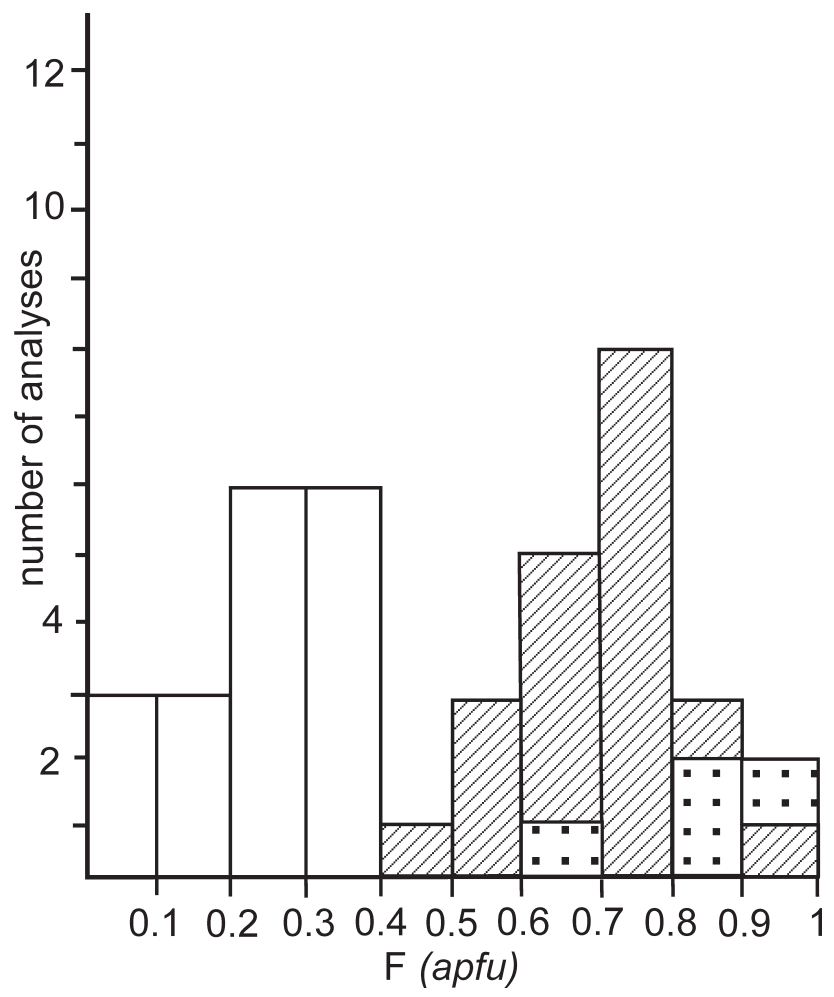

Table 2 Representative microprobe analyses of clintonite.

\begin{tabular}{|c|c|c|c|c|c|c|c|c|c|c|c|c|}
\hline & $\begin{array}{r}\text { CHL-I } \\
\text { Cli }\end{array}$ & $\begin{array}{r}\text { CHL-I } \\
\text { Cli }\end{array}$ & $\begin{array}{c}\text { CHL-I S } \\
\text { Cli }\end{array}$ & $\begin{array}{c}\text { SOKP-I } \\
\mathrm{Cli}\end{array}$ & $\begin{array}{r}\text { SOKP-I } \\
\mathrm{Cli}\end{array}$ & $\begin{array}{c}\text { SOKP-IS } \\
\text { Cli }\end{array}$ & $\begin{array}{l}\text { OKP-II S } \\
\text { Cli }\end{array}$ & $\begin{array}{r}\text { OKP-II } \\
\text { Cli }\end{array}$ & $\begin{array}{r}\text { TAS-I } \\
\text { Cli }\end{array}$ & $\begin{array}{r}\text { TAS-I } \\
\text { Cli }\end{array}$ & $\begin{array}{r}\text { TAS-II } \\
\text { Cli }\end{array}$ & $\begin{array}{r}\text { TAS-II } \\
\text { Cli } \\
\end{array}$ \\
\hline $\mathrm{SiO}_{2}$ & 19.57 & 19.68 & 19.99 & 20.19 & 20.00 & 20.24 & 20.44 & 19.91 & 20.04 & 20.54 & 21.00 & 20.71 \\
\hline $\mathrm{TiO}_{2}$ & 0.17 & 0.19 & 0.14 & 0.06 & 0.04 & 0.07 & 0.04 & 0.05 & 0.06 & 0.07 & 0.09 & 0.09 \\
\hline $\mathrm{Al}_{2} \mathrm{O}_{3}$ & 39.51 & 39.32 & 38.69 & 38.36 & 39.16 & 38.37 & 38.55 & 37.42 & 37.77 & 38.15 & 37.02 & 36.89 \\
\hline $\mathrm{Cr}_{2} \mathrm{O}_{3}$ & 0.03 & 0.06 & 0.02 & 0.04 & 0.03 & 0.03 & 0.06 & 0.03 & 0.03 & 0.05 & 0.01 & b.d. \\
\hline $\mathrm{FeO}$ & 0.72 & 0.80 & 0.70 & 0.48 & 0.57 & 0.35 & 0.49 & 0.41 & 1.01 & 0.91 & 1.07 & 1.08 \\
\hline MgO & 21.81 & 21.94 & 22.40 & 22.82 & 22.34 & 22.84 & 22.34 & 22.49 & 22.29 & 22.57 & 22.44 & 22.45 \\
\hline $\mathrm{CaO}$ & 13.23 & 13.12 & 13.31 & 13.09 & 13.29 & 13.29 & 13.57 & 13.23 & 12.81 & 12.9 & 12.83 & 12.81 \\
\hline $\mathrm{Na}_{2} \mathrm{O}$ & 0.18 & 0.31 & 0.18 & 0.20 & 0.13 & 0.21 & 0.13 & 0.13 & 0.28 & 0.35 & 0.33 & 0.34 \\
\hline $\mathbf{K}_{2} \mathbf{O}$ & 0.02 & 0.02 & 0.02 & 0.02 & 0.02 & 0.01 & b.d. & 0.01 & 0.02 & 0.03 & 0.03 & 0.02 \\
\hline $\mathrm{H}_{2} \mathrm{O}$ * & 3.51 & 3.52 & 3.46 & 3.47 & 3.82 & 3.67 & 3.41 & 3.51 & 3.48 & 3.43 & 3.38 & 3.15 \\
\hline F & 1.60 & 1.59 & 1.73 & 1.71 & 0.99 & 1.29 & 1.86 & 1.46 & 1.57 & 1.81 & 1.84 & 2.28 \\
\hline $\mathbf{O}=\mathbf{F}$ & -0.67 & -0.67 & -0.73 & -0.72 & -0.42 & -0.54 & -0.78 & -0.61 & -0.66 & -0.76 & -0.77 & -0.96 \\
\hline TOTAL & 99.68 & 99.89 & 99.91 & 99.72 & 99.98 & 99.83 & 100.11 & 98.04 & 98.70 & 100.05 & 99.27 & 98.86 \\
\hline $\mathrm{Si}^{4+}$ & 2.747 & 2.758 & 2.800 & 2.829 & $\begin{array}{l}9.794 \\
\end{array}$ & $\begin{array}{l}4 \quad 2.832 \\
\end{array}$ & 2.854 & 2.839 & 2.843 & 2.873 & 2.961 & 2.936 \\
\hline $\mathrm{Al}^{\mathrm{IV}}$ & 5.253 & 5.242 & 5.200 & 5.171 & $1 \quad 5.206$ & $\begin{array}{ll}6 & 5.168\end{array}$ & 5.146 & 5.161 & 5.157 & 5.127 & 5.039 & 5.064 \\
\hline $\mathbf{A l}^{\mathrm{VI}}$ & 1.282 & 1.253 & 1.188 & $\begin{array}{ll}3 & 1.165\end{array}$ & $5 \quad 1.241$ & $\begin{array}{ll}1 & 1.159\end{array}$ & 1.199 & 1.129 & 1.159 & 1.162 & 1.113 & 1.099 \\
\hline $\mathrm{Ti}^{4+}$ & 0.018 & 0.020 & 0.015 & $5 \quad 0.006$ & $\begin{array}{ll}6 & 0.004\end{array}$ & $\begin{array}{ll}4 & 0.007\end{array}$ & 0.004 & 0.005 & 0.006 & 0.007 & 0.010 & 0.010 \\
\hline $\mathrm{Cr}^{3+}$ & 0.003 & 0.007 & 0.002 & $2 \quad 0.004$ & $\begin{array}{l}4 \quad 0.003 \\
\end{array}$ & $\begin{array}{ll}3 & 0.003\end{array}$ & 0.007 & 0.003 & 0.003 & 0.006 & 0.001 & - \\
\hline $\mathrm{Fe}^{2+}{ }_{\text {tot }}$ & 0.085 & 0.094 & 0.082 & $2 \quad 0.056$ & $\begin{array}{ll}6 & 0.067\end{array}$ & $\begin{array}{ll}7 & 0.041\end{array}$ & 0.057 & 0.049 & 0.120 & 0.106 & 0.126 & 0.128 \\
\hline $\mathbf{M g}^{2+}$ & 4.563 & 4.584 & 4.678 & 3.768 & $8 \quad 4.652$ & $2 \quad 4.764$ & 4.651 & 4.782 & 4.715 & 4.707 & 4.717 & 4.744 \\
\hline $\mathrm{Ca}^{2+}$ & 1.989 & 1.970 & 1.998 & 1.965 & $\begin{array}{ll}5 & 1.989\end{array}$ & $9 \quad 1.992$ & 2.030 & 2.022 & 1.947 & 1.933 & 1.938 & 1.945 \\
\hline $\mathrm{Na}^{+}$ & 0.049 & 0.084 & 0.049 & 0.054 & $4 \quad 0.035$ & $\begin{array}{ll}5 & 0.057\end{array}$ & 0.035 & 0.036 & 0.077 & 0.095 & 0.090 & 0.093 \\
\hline $\mathbf{K}^{+}$ & 0.004 & 0.004 & 0.004 & 0.004 & $\begin{array}{l}4 \quad 0.004 \\
4\end{array}$ & $\begin{array}{ll}4 & 0.002\end{array}$ & - & - & 0.004 & 0.005 & 0.005 & 0.004 \\
\hline $\mathbf{H}^{+}$ & 3.290 & 3.295 & 3.233 & 3.242 & $2 \quad 3.563$ & $\begin{array}{l}3 \quad 3.429 \\
\end{array}$ & 3.179 & 3.341 & 3.296 & 3.199 & 3.179 & 2.978 \\
\hline$F^{-}$ & 0.710 & 0.705 & 0.767 & 0.758 & $8 \quad 0.437$ & $\begin{array}{ll}7 & 0.571\end{array}$ & 0.821 & 0.659 & 0.704 & 0.801 & 0.821 & 1.022 \\
\hline $\mathrm{O}^{2-}$ & 23.29 & 23.295 & 23.233 & 23.242 & 223.563 & $\begin{array}{ll}3 \quad 23.429\end{array}$ & 23.179 & 23.341 & 23.296 & 23.199 & 23.179 & 22.978 \\
\hline CATSUM & 15.993 & 16.015 & 16.016 & 16.023 & 315.996 & $\begin{array}{ll}6 & 16.025\end{array}$ & 15.983 & 16.028 & 16.031 & 16.022 & 16.001 & 16.022 \\
\hline AN SUM & 24 & 24 & 24 & 24 & 24 & 24 & 24 & 24 & 24 & 24 & 24 & 24 \\
\hline $\mathbf{X}_{\mathrm{F}}$ & 0.18 & 0.18 & 0.19 & 0.19 & 0.11 & 0.14 & 0.21 & 0.17 & 0.18 & 0.20 & 0.21 & 0.26 \\
\hline
\end{tabular}


the possibility of presence of submicroscopic intergrowth of chondrodite with humite (cf. Rice 1980, Satish-Kumar - Niimi 1998). Representative chemical data of chondrodite are given in Table 3.

Other minerals

Phlogopite has high $\mathrm{X}_{\mathrm{Mg}}=0.96-0.99$ and high Al (up to $2.726 \mathrm{apfu})$, low content of Ti ( $\leq 0.033 \mathrm{apfu}), \mathrm{Fe}(\leq 0.229$ apfu), $\mathrm{Mn}(\leq 0.005 \mathrm{apfu})$ and $\mathrm{Na}(=0.294 \mathrm{apfu})$, concentration of $\mathrm{Ba}$ is below detection limit (Table 4). Elevated contents of F $\left(\mathrm{X}_{\mathrm{F}}=0.20-0.39\right)$ are typical. Pargasite (to rare edenite) shows $\mathrm{X}_{\mathrm{Mg}}=0.92-0.94$, variable contents of Al (1.927-2.732 apfu), Ti (0.029-0.098 apfu) and $\mathrm{Fe}(0.159-0.354 \mathrm{apfu}), \mathrm{Mn}$ is always close to the detection limit. Pargasite from Cíhalín and Sokolí is relatively rich in $\mathrm{K}(\leq 0.321 \mathrm{apfu})$, locally with elevated content of $\mathrm{F}\left(\mathrm{X}_{\mathrm{F}}=0.39-0.61\right)$ (Table 4). Diopside is close to the end-member composition with $\mathrm{X}_{\mathrm{Mg}}=0.98-1.0$. Spinel exhibits moderate $\mathrm{X}_{\mathrm{Mg}}=0.78-0.90$, slightly elevated contents of $\mathrm{Fe}(\leq 0.178 \mathrm{apfu})$ and $\mathrm{Zn}(\leq 0.043$ apfu), with $\mathrm{Mn}$ and $\mathrm{Cr}$ close to the detection limits (Table 4). This Fe-rich spinel from locality Tasov has similar composition as early core of euhedral spinel from

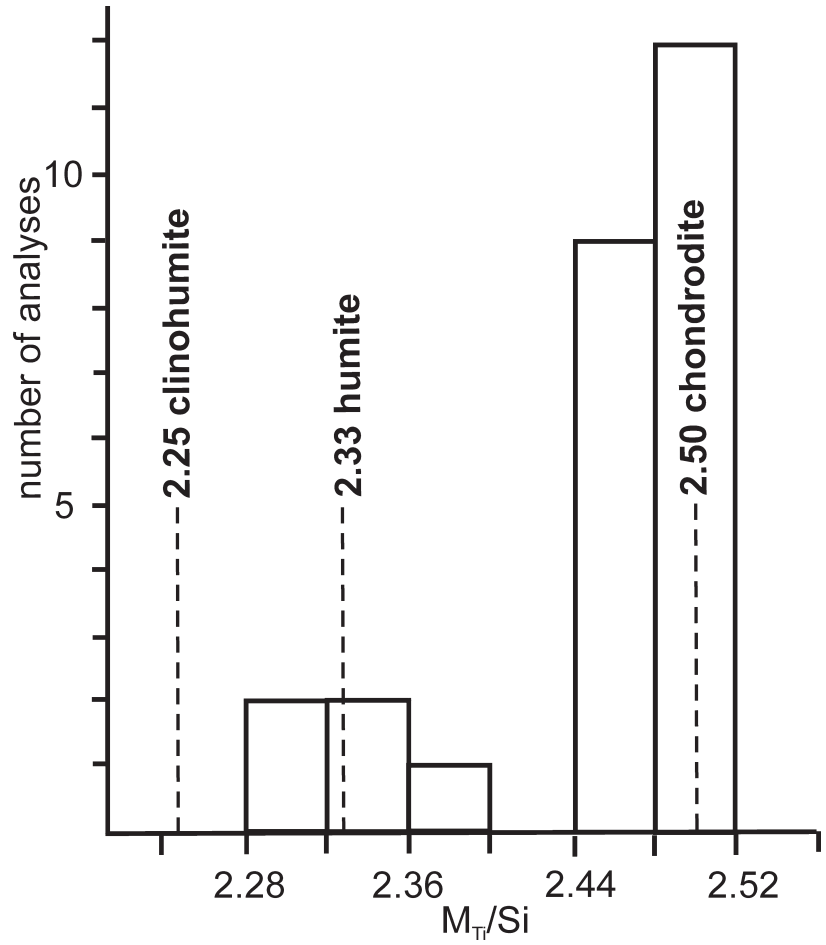

Fig. 5 Diagram showing $\mathrm{M}_{\mathrm{Ti}} / \mathrm{Si}$ ratio in studied chondrodite.

Table 3 Representative microprobe analyses of chondrodite and chlorite II.

\begin{tabular}{|c|c|c|c|c|c|c|c|c|}
\hline & $\begin{array}{r}\text { CHL-I } \\
\text { Chn* }\end{array}$ & $\begin{array}{r}\text { SOKP-I } \\
\text { Chn }\end{array}$ & $\begin{array}{r}\text { SOKP-II } \\
\text { Chn }\end{array}$ & $\begin{array}{r}\text { TAS-I } \\
\text { Chn }\end{array}$ & $\begin{array}{r}\text { TAS-II } \\
\text { Chn }\end{array}$ & $\begin{array}{r}\text { CHL-I } \\
\text { Chl II }\end{array}$ & $\begin{array}{r}\text { SOKP-II } \\
\text { Chl II } \\
\end{array}$ & $\begin{array}{l}\text { TAS-I } \\
\text { ChI II }\end{array}$ \\
\hline $\mathrm{SiO}_{2}$ & 37.28 & 34.87 & 35.25 & 35.32 & 34.89 & 32.16 & 31.17 & 30.82 \\
\hline $\mathrm{TiO}_{2}$ & 0.59 & 0.30 & 0.38 & 0.27 & 0.31 & 0.07 & 0.02 & 0.05 \\
\hline $\mathrm{Al}_{2} \mathrm{O}_{3}$ & b.d. & b.d. & b.d. & b.d. & b.d. & 19.66 & 21.51 & 20.87 \\
\hline $\mathrm{FeO}$ & 3.51 & 2.22 & 2.26 & 2.75 & 4.65 & 1.55 & 0.98 & 1.70 \\
\hline MnO & 0.07 & 0.04 & 0.04 & 0.06 & 0.16 & b.d. & b.d. & b.d. \\
\hline MgO & 55.21 & 56.98 & 57.17 & 56.53 & 54.99 & 34.09 & 33.68 & 33.31 \\
\hline $\mathrm{CaO}$ & 0.05 & 0.02 & b.d. & 0.07 & 0.04 & 0.18 & 0.03 & 0.03 \\
\hline $\mathrm{H}_{2} \mathrm{O}$ * & 2.79 & 1.92 & 2.11 & 1.72 & 1.97 & 12.71 & 12.66 & 12.27 \\
\hline $\mathbf{F}$ & 5.42 & 6.98 & 6.66 & 7.46 & 6.83 & 0.56 & 0.68 & 1.17 \\
\hline $\mathrm{O}=\mathrm{F}$ & -2.28 & -2.94 & -2.80 & -3.14 & -2.88 & -0.24 & -0.29 & -0.49 \\
\hline TOTAL & 102.63 & 100.39 & 101.08 & 101.04 & 100.96 & 100.95 & 100.52 & 99.73 \\
\hline $\mathrm{Si}^{4+}$ & 2.087 & 1.998 & 2.005 & 2.015 & 2.009 & 2.969 & 2.880 & 2.882 \\
\hline $\mathbf{T i}^{4+}$ & 0.025 & 0.013 & 0.016 & 0.012 & 0.013 & 0.005 & 0.001 & 0.004 \\
\hline $\mathbf{A l}^{3+}$ & - & - & - & - & - & 2.139 & 2.342 & 2.300 \\
\hline $\mathrm{Fe}^{2+}$ tot & 0.164 & 0.106 & 0.107 & 0.131 & 0.224 & 0.120 & 0.076 & 0.133 \\
\hline $\mathbf{M n}^{2+}$ & 0.003 & 0.002 & 0.002 & 0.003 & 0.008 & - & - & - \\
\hline $\mathrm{Mg}^{2+}$ & 4.607 & 4.868 & 4.848 & 4.808 & 4.721 & 4.692 & 4.639 & 4.643 \\
\hline $\mathrm{Ca}^{2+}$ & 0.003 & 0.001 & - & 0.004 & 0.002 & 0.018 & 0.003 & 0.003 \\
\hline $\mathbf{H}^{+}$ & 1.041 & 0.735 & 0.802 & 0.654 & 0.756 & 7.826 & 7.800 & 7.652 \\
\hline $\mathbf{F}^{-}$ & 0.959 & 1.265 & 0.198 & 1.346 & 1.244 & 0.164 & 0.199 & 0.346 \\
\hline $\mathrm{O}^{2-}$ & 9.041 & 8.735 & 8.802 & 8.654 & 8.756 & 17.826 & 17.800 & 17.652 \\
\hline CATSUM & 6.889 & 6.989 & 6.979 & 6.973 & 6.977 & 9.964 & 9.947 & 9.967 \\
\hline AN SUM & 10 & 10 & 10 & 10 & 10 & 18 & 18 & 18 \\
\hline $\mathbf{X}_{\mathrm{F}}$ & 0.48 & 0.63 & 0.60 & 0.67 & 0.61 & & & \\
\hline
\end{tabular}

* including probable humite 
Table 4 Representative microprobe analyses of pargasite, phlogopite, diopside and spinel.

\begin{tabular}{|c|c|c|c|c|c|c|c|c|c|c|c|}
\hline & $\begin{array}{r}\text { CHL-I } \\
\text { Prg }\end{array}$ & $\begin{array}{r}\text { SOKP-I } \\
\text { Prg }\end{array}$ & $\begin{array}{r}\text { TAS-II } \\
\text { Prg }\end{array}$ & $\begin{array}{r}\text { CHL-I } \\
\text { Phl }\end{array}$ & $\begin{array}{r}\text { SOKP-I } \\
\text { Phl }\end{array}$ & $\begin{array}{r}\text { SOKP-II } \\
\text { Phl }\end{array}$ & $\begin{array}{r}\text { TAS-II } \\
\text { Phl }\end{array}$ & $\begin{array}{r}\text { CHL-I } \\
\text { Di }\end{array}$ & $\begin{array}{r}\text { SOKP-I } \\
\text { Spl }\end{array}$ & $\begin{array}{c}\text { AS-I cor } T \\
\text { Spl }\end{array}$ & $\begin{array}{r}\text { S-I rim } \\
\text { Spl }\end{array}$ \\
\hline $\mathrm{SiO}_{2}$ & 43.42 & 42.48 & 43.15 & 41.12 & 41.31 & 40.79 & 42.06 & 55.31 & b.d. & b.d. & b.d. \\
\hline $\mathrm{TiO}_{2}$ & 0.92 & 0.27 & 0.81 & 0.28 & 0.14 & 0.19 & 0.28 & 0.05 & b.d. & b.d. & b.d. \\
\hline $\mathrm{Al}_{2} \mathrm{O}_{3}$ & 15.05 & 15.06 & 14.23 & 15.85 & 15.37 & 15.68 & 14.87 & 0.17 & 69.64 & 67.46 & 67.45 \\
\hline $\mathrm{Cr}_{2} \mathrm{O}_{3}$ & b.d. & b.d. & b.d. & b.d. & 0.04 & 0.03 & b.d. & b.d. & 0.19 & b.d. & b.d. \\
\hline $\mathrm{Fe}_{2} \mathrm{O}_{3}$ & & & & & & & & & 1.07 & 2.13 & 1.45 \\
\hline FeO & 1.37 & 2.43 & 2.97 & 1.02 & 0.77 & 0.67 & 1.97 & 0.85 & 3.62 & 7.42 & 8.59 \\
\hline MnO & 0.03 & b.d. & b.d. & 0.03 & 0.04 & 0.02 & b.d. & 0.02 & 0.04 & b.d. & b.d. \\
\hline $\mathrm{ZnO}$ & b.d. & b.d. & b.d. & b.d. & b.d. & b.d. & b.d. & b.d. & 1.44 & 1.66 & 2.32 \\
\hline MgO & 18.81 & 20.28 & 18.70 & 25.74 & 27.35 & 27.04 & 25.92 & 18.00 & 25.11 & 22.22 & 21.06 \\
\hline $\mathrm{CaO}$ & 13.93 & 13.66 & 13.48 & b.d. & b.d. & b.d. & b.d. & 25.98 & b.d. & b.d. & b.d. \\
\hline $\mathrm{Na}_{2} \mathrm{O}$ & 2.21 & 1.62 & 3.18 & 0.84 & 0.22 & 0.21 & 1.09 & 0.05 & b.d. & b.d. & b.d. \\
\hline $\mathrm{K}_{2} \mathrm{O}$ & 1.50 & 1.23 & 0.96 & 10.06 & 10.43 & 10.49 & 9.47 & b.d. & b.d. & b.d. & b.d. \\
\hline $\mathrm{H}_{2} \mathrm{O} *$ & 1.22 & 1.10 & 1.47 & 2.66 & 3.18 & 3.34 & 3.29 & & & & \\
\hline $\mathrm{F}$ & 1.88 & 2.12 & 1.25 & 3.43 & 2.40 & 2.01 & 2.16 & & & & \\
\hline $\mathbf{O}=\mathbf{F}$ & -0.79 & -0.89 & -0.53 & -1.44 & -1.01 & -0.85 & -0.91 & & & & \\
\hline TOTAL & 99.55 & 99.35 & 99.80 & 99.58 & 100.24 & 99.62 & 100.2 & 100.43 & 101.12 & 100.89 & 100.88 \\
\hline $\mathrm{Si}^{4+}$ & 6.162 & 6.059 & 6.158 & 5.756 & 5.739 & 5.701 & 5.849 & 1.994 & - & - & - \\
\hline $\mathbf{T i}^{4+}$ & 0.098 & 0.029 & 0.087 & 0.029 & 0.015 & 0.020 & 0.029 & 0.001 & - & - & - \\
\hline $\mathbf{A l}^{3+}$ & 2.517 & 2.532 & 2.393 & 2.615 & 2.516 & 2.583 & 2.437 & 0.007 & 1.976 & 1.960 & 1.973 \\
\hline $\mathrm{Cr}^{3+}$ & - & - & - & - & 0.004 & 0.003 & - & - & 0.004 & - & - \\
\hline $\mathrm{Fe}^{3+}$ & & & & & & & & & 0.019 & 0.040 & 0.027 \\
\hline $\mathrm{Fe}^{2+}$ & 0.163 & 0.290 & 0.354 & 0.119 & 0.089 & 0.078 & 0.229 & 0.026 & 0.073 & 0.153 & 0.178 \\
\hline $\mathrm{Mn}^{2+}$ & 0.004 & - & - & 0.004 & 0.005 & 0.002 & 0 & 0.001 & 0.001 & - & - \\
\hline $\mathrm{Zn}^{2+}$ & - & - & - & - & - & - & - & - & 0.026 & 0.030 & 0.043 \\
\hline $\mathrm{Mg}^{2+}$ & 3.980 & 4.312 & 3.978 & 5.371 & 5.664 & 5.634 & 5.373 & 0.967 & 0.901 & 0.817 & 0.779 \\
\hline $\mathrm{Ca}^{2+}$ & 2.018 & 2.088 & 2.061 & - & - & - & - & 1.003 & - & - & - \\
\hline $\mathbf{N a}^{+}$ & 0.608 & 0.448 & 0.880 & 0.228 & 0.059 & 0.057 & 0.294 & 0.003 & - & - & - \\
\hline $\mathbf{K}^{+}$ & 0.272 & 0.224 & 0.175 & 1.797 & 1.848 & 1.870 & 1.680 & - & - & - & - \\
\hline $\mathbf{H}^{+}$ & 1.156 & 1.044 & 1.397 & 2.481 & 2.946 & 3.111 & 3.050 & - & - & - & - \\
\hline $\mathbf{F}^{-}$ & 0.844 & 0.956 & 0.564 & 1.519 & 1.054 & 0.889 & 0.950 & - & - & - & - \\
\hline $\mathrm{O}^{2-}$ & 23.156 & 23.044 & 23.397 & 22.481 & 22.946 & 23.111 & 23.050 & - & - & - & - \\
\hline CATSUM & 15.921 & 15.982 & 16.086 & 15.919 & 15.94 & 15.949 & 15.891 & 4 & 3 & 3 & 3 \\
\hline AN SUM & 24 & 24 & 24 & 24 & 24 & 24 & 24 & 6 & 4 & 4 & 4 \\
\hline
\end{tabular}

dolomite marbles with the assemblage $\mathrm{Fo}+\mathrm{Chu}+\mathrm{Spl}$ in Horní Libochová (Novák 1988). Both chlorite I and chlorite II are colourless. Only chlorite II was analysed having high $\mathrm{X}_{\mathrm{Mg}}=0.97-0.99$ with $\mathrm{Fe}(\leq 0.120 \mathrm{apfu})$ and $\mathrm{Al}$ contents 2.004-2.342 apfu. Chlorite II locally contains small amounts of $\mathrm{F} \leq 0.346$ apfu (Table 3 ).

\section{Discussion}

\subsection{Textural relations and mineral reactions}

Textural relations in clintonite-bearing chondrodite marbles enable to recognize three stages, which are characterized by the mineral assemblages (calcite + dolomite are always present): (i) pargasite+phlogopite+diopside(?); (ii) chondrodite+clintonite+chlorite I or spinel; (iii) chlorite II. Spinel as well as chlorite I, both formed by replacement of phlogopite (or pargasite) are locally absent. Position of diopside is not clear, because it is always enclosed in calcite and its contact with any other silicate mineral was not found. This sequence of mineral assemblages is very similar to that known from dolomite marbles in this region, where the following mineral assemblages were recognized in the individual stages $(\mathrm{Cal}+\mathrm{Dol}$ always present): (i) tremolite+phlogopite+pargasite; (ii) forsterite+clinohumite+spinel+chlorite I; (iii) chlorite II (cf. Novák 1988, 1989, Novák - Houzar 1996, Houzar 2004).

Mineral reactions producing clintonite in marbles involve calcite, clinopyroxene (diopside), forsterite and monticellite as reactants, spinel is considered as a source 
of Al (Bucher-Nurminen 1975, Rice 1979, Ulmer 1983).

The following reactions were given:

(1) $\mathrm{Fo}+\mathrm{Cpx}+\mathrm{Spl}+\mathrm{Cal}+\mathrm{H}_{2} \mathrm{O}=\mathrm{Cli}+\mathrm{CO}_{2}$

(2) $\mathrm{Cpx}+\mathrm{Spl}+\mathrm{Cal}+\mathrm{H}_{2} \mathrm{O}=\mathrm{Cli}+\mathrm{Fo}+\mathrm{CO}_{2}$

The reaction (2) strongly depends on $\mathrm{X}_{\mathrm{CO} 2}$, particularly for $\mathrm{T}>550{ }^{\circ} \mathrm{C}$ (Rice 1979). Bucher-Nurminen (1975) discussed a possibility of clintonite production by dealkalization of phlogopite and pargasite according to the reactions: $\mathrm{Na}_{2} \mathrm{O}$

(3) $\mathrm{Cal}+\mathrm{Phl}+\mathrm{Prg}=\mathrm{Cli}+\mathrm{Di}+\mathrm{CO}_{2}+\mathrm{H}_{2} \mathrm{O}+\mathrm{K}_{2} \mathrm{O}+$

(4) $\mathrm{Cal}+\mathrm{Phl}+\mathrm{Spl}+\mathrm{H}_{2} \mathrm{O}=\mathrm{Cli}+\mathrm{Fo}+\mathrm{CO}_{2}+\mathrm{K}_{2} \mathrm{O}$

However, dolomite as a typical mineral in these assemblages and typical reactant in prograde carbonate + silicate reactions (see e.g. Skippen 1974) is not involved in the reactions (1) to (4). Moreover, participation of minerals with elevated concentrations of $\mathrm{F}$ has not been discussed as well. Consequently, the reactions given above cover only a small part of natural mineral assemblages found in marbles.

The examined localities differ in their mineral assemblages and textural relations from the above examples; hence, new reactions (detailed stoichiometry is not given) have been suggested. At the Číhalín locality, textural relations indicate the reaction:

(5) $\mathrm{Dol}+\mathrm{Phl}+\mathrm{Di}+\mathrm{H}_{2} \mathrm{O}=\mathrm{Cli}+\mathrm{Chn}+\mathrm{Chl} \mathrm{I}+\mathrm{Cal}$ $+\mathrm{CO}_{2}+\mathrm{K}_{2} \mathrm{O}$

At Sokolí and Tasov, the presence of pargasite and phlogopite and their textural relations suggest the reactions:

(6) $\mathrm{Dol}+\mathrm{Prg}+\mathrm{H}_{2} \mathrm{O}=\mathrm{Cli}+\mathrm{Chn}+\mathrm{Cal}+\mathrm{CO}_{2}+\mathrm{Na}_{2} \mathrm{O}$ $\pm \mathrm{K}_{2} \mathrm{O}$

(7) $\mathrm{Dol}+\mathrm{Phl}+\mathrm{H}_{2} \mathrm{O}=\mathrm{Cli}+\mathrm{Chn}+\mathrm{Cal}+\mathrm{CO}_{2}+\mathrm{K}_{2} \mathrm{O}$

(8) $\mathrm{Dol}+\mathrm{Phl}$ (and/or Prg) $+\mathrm{SiO}_{2 \mathrm{aq}}+\mathrm{F}=\mathrm{Cli}+\mathrm{Chn}+$ $\mathrm{Spl}+\mathrm{Cal}+\mathrm{CO}_{2}+\mathrm{K}_{2} \mathrm{O} \pm \mathrm{Na}_{2} \mathrm{O}$

The abundance of chondrodite (up to $\sim 25$ vol. \%), high chondrodite/clintonite ratios and isotopic composition of calcite support introduction of $\mathrm{H}_{2} \mathrm{O}$, Si and very likely also $\mathrm{F}$ into the system, whereas $\mathrm{Na}$ and $\mathrm{K}$ were removed. Based on detailed study of reaction textures, similar dealkalization reactions producing chlorite and/or spinel instead of clintonite, and forsterite instead of chondrodite were described for amphibole and phlogopite (Kretz 1980, Bucher-Nurminen 1982, Novák 1989). However, they were not tested experimentally and the breakdown of pargasite and phlogopite is still unclear; perhaps a high acidity of fluids may have operated (cf. Bucher-Nurminen 1982).

Retrograde chlorite II, occurring exclusively at the contact between chondrodite and clintonite, formed by the reaction:

(9) $\mathrm{Cli}+\mathrm{Chn}+\mathrm{CO}_{2}+\mathrm{H}_{2} \mathrm{O}=\mathrm{Chl}+\mathrm{Cal}$

The reactions (7) and (9) are very similar to those described by Novák (1989) and Novák - Houzar (1996) for dolomite marbles in the Strážek Moldanubicum, characterized by the presence of forsterite and/or clinohumite instead of chondrodite and spinel and/or chlorite I instead of clintonite.

\subsection{Role of fluorine in mineral reactions}

The mineral reactions producing clintonite in marbles were only exceptionally studied experimentally (Rice 1979). The assemblage chondrodite+clintonite is rare in nature and it has not been studied to date. Moreover, the reactions observed in the studied region are quite different from those examined by Rice (1979). Consequently, we can estimate PT $(\mathrm{X})$ conditions of formation of the assemblage chondrodite+clintonite only from analogy with associated dolomite marbles with the assemblage $\mathrm{Fo}+\mathrm{Chu}+\mathrm{Spl}+\mathrm{Chl}$. Nevertheless, the estimated $\mathrm{T} \sim 620$ $730{ }^{\circ} \mathrm{C}$ for $\mathrm{P} \sim 2-4 \mathrm{kbar}$ for these marbles are in agreement with the stability field of clintonite and chondrodite (Olesch - Seifert 1976, Rice 1979, 1980).

The mineral assemblages and reactions described above may suggest two distinct ways of clintonite formation controlled chiefly by activity of F. If the newly formed mineral assemblage shows low amount of chondrodite and clintonite, and low chondrodite/clintonite ratio, replaced phlogopite and/or pargasite possibly provided enough $\mathrm{F}$ for formation of the both F-bearing minerals, e.g., according to the reaction (5) and perhaps also reactions (6) and (7); influx of F into the system is not necessary. Novák (1988) found such a relation in a newly formed assemblage $\mathrm{Fo}+\mathrm{Chu}+\mathrm{Spl}+\mathrm{Cal}$ replacing phlogopite and dolomite at locality Studnice in the Strážek Moldanubicum. Nevertheless, the most widespread textural relations, abundance of chondrodite (locally up to 25 vol. \%), high chondrodite/clintonite ratio as well as the isotopic compositions of calcite from some samples at Sokolí and Tasov strongly suggest influx of F in external fluids, where the reaction (8) and perhaps also reactions (6) and (7) operated. Consequently, F-rich clintonite $\left(\mathrm{X}_{\mathrm{F}}=0.11-0.26\right)$ associated with chondrodite crystallized from external fluids introducing $\mathrm{F}, \mathrm{Si}$ and $\mathrm{H}_{2} \mathrm{O}$ but perhaps at relatively high $\mathrm{X}_{\mathrm{CO} 2}<0.6$. The modal composition and textural relations of the assemblage chondrodite + clintonite suggest dominance of the crystallization in an open system with influx of $\mathrm{F}$ at most localities, relative to crystallization in a closed system. The latter case may correspond only to marble domains with a low chondrodite content.

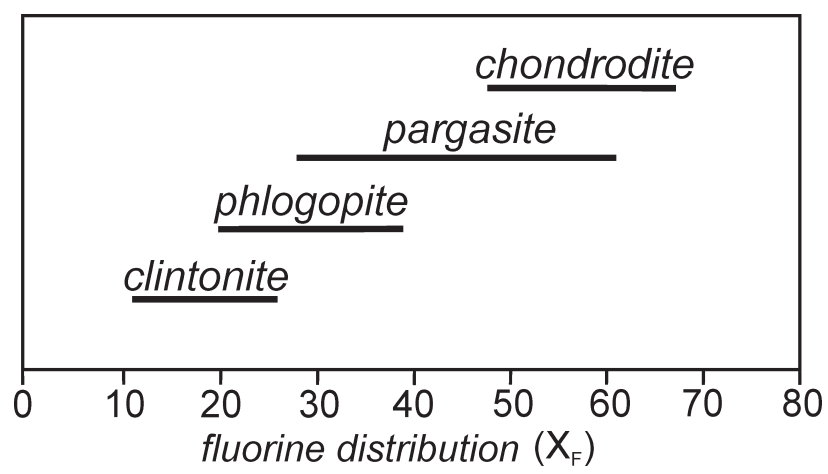

Fig. 6 Distribution of $F$ in the minerals from the studied chondrodite marbles. 
Partitioning of $\mathrm{F}$ into the individual minerals: $\mathrm{X}_{\mathrm{F}}=$ $\mathrm{Cli}_{(0.11-0.26)}<\mathrm{Phl}{ }_{(0.20-0.39)}<\mathrm{Prg}{ }_{(0.28-0.61)}<\mathrm{Chn}{ }_{(0.45-0.67)}$ (Fig. 6) is similar to that found in marbles from the Franklin Marble, Orange County, USA (Kearns et al. 1980). Disregarding the fact that the examined clintonite is F-poor relative to the other associated minerals, it is characterized by the highest concentrations of $\mathrm{F}$ ever recorded in this mineral $\left(\mathrm{X}_{\mathrm{F}}=0.11-0.26 ; 1.13-2.28\right.$ wt. \% F $)$. Similar assemblages and high $\mathrm{F}$ contents in clintonite were found in marbles at localities Amity, Edenville and Warwick (Orange County, New York, USA; $\left(\mathrm{X}_{\mathrm{F}}=0.17-\right.$ 0.23 ; $1.45-2.05$ wt. \% F; MacKinney et al. 1988). This is in contradiction with a quite low amount of $\mathrm{F}$ in associated chondrodite $\left(\mathrm{X}_{\mathrm{F}}=0.45-0.67\right)$ relative to chondrodite from other localities and experimental study (Rice 1980, 1979).

\section{Conclusions}

Formation of clintonite is very likely related to HT/LP metamorphism in the aureole of the Třebíč Pluton. Bucher - Frey (1994) considered clintonite a typomorphic mineral in the mineral assemblages $\mathrm{Cal}+\mathrm{Fo}+\mathrm{Chu}+\mathrm{Spl}+\mathrm{Cli}$ and $\mathrm{Cal}+\mathrm{Fo}+\mathrm{Di}+\mathrm{Spl}+\mathrm{Cli}$ located in contact aureoles of granite plutons. However, why clintonite occurs only in the chondrodite marbles but it is absent in associated dolomite marbles with the assemblage $\mathrm{Cal}+\mathrm{Fo}+\mathrm{Chu}+\mathrm{Spl}+\mathrm{Chl}$, very similar to those described by Bucher - Frey (1994), is unclear. Origin of clintonite seems to be related to elevated activity of $\mathrm{F}$ indicated also by abundant chondrodite. Its composition with $\mathrm{X}_{\mathrm{F}}=$ $0.45-0.67$ suggests elevated activity of $F$ but also variable and high $\mathrm{X}_{\mathrm{CO} 2}<0.6$ (see Rice 1980) relative to rather low $\mathrm{X}_{\mathrm{CO} 2}<0.4$ derived from clinohumite composition and mineral relations in associated dolomite marbles with clinohumite (Novák - Houzar 1996). Activity of F seems to be the main factor controlling formation of clintonite besides general factors such as low $\mathrm{Si} / \mathrm{Al}$ ratio and a low activity of $\mathrm{K}$ and $\mathrm{Na}$.

All localities of chondrodite marbles occur within clinohumite zone developed around the Třebíč Pluton. They are enclosed in migmatites and the distance of the individual localities from the nearest outcrop of durbachite rocks at the present surface varies from $\sim 0.2$ to $\sim 3 \mathrm{~km}$. Along with the examined Moravian localities, clintonite was recently discovered by the authors at Kamenné doly quarry near Písek, southern Bohemia. This chondrodite marble forms enclave, $\sim 4 \mathrm{~m}$ in diameter, enclosed in durbachite. It exhibits the assemblage $\mathrm{Cal}+\mathrm{Cli}+\mathrm{Chn}+\mathrm{Prg}+\mathrm{Spl}+\mathrm{Chl} \mathrm{II}$, very similar to those described in chondrodite marbles around the Třebíč Pluton. The presence of marble enclave in durbachite supports contact-metamorphic origin of clintonite in the inner part of aureole of the Třebíč Pluton, disregarding large distance of some clintonite localities from the nearest durbachite outcrop. However, as the Třebíc Pluton forms a tabular body (Rejl - Sedlák 1987), the distance of chondrodite marbles from the nearest durbachite outlier, as estimated in geological maps, may be incorrect. The Tasov borehole documented tabular durbachite bodies overlying chondrodite marbles and indicates that similar overlying bodies of durbachite may have been removed by erosion at the other studied localities.

Acknowledgements. The authors thank D. Buriánek and S. Vrána for helpful reviews that improved the manuscript. We also thank J. Cempírek for helpful comments and technical assistance. The work was supported by grants MK 00009486201 for SH and MSM 0021622412 for $\mathrm{MN}$.

Submitted October 27, 2006

\section{References}

Ackermand, D. - Herd, R. K. - Windley, B. F. (1986): Clintonite of regional metamorphic origin, along the margin of the Fiskenaesset complex, West Greenland. - N. Jb. Miner. Abh., 155: 39-51.

Alietti, E. - Brigatti, M. F. - Poppi, L. (1997): Clintonite-1M: Crystal chemistry and its relationships to closely associated Al-rich phlogopite. - Amer. Mineral., 82: 936-945.

Bucher-Nurminen, K. (1976): Occurrence and chemistry of xantophyllite in roof pendants of the Bergell Granite, Sondrio, Northern Italy. Schwiez. Mineral. Petrogr. Mitt., 56: 413-426.

- (1982): Mechanism of mineral reactions inferred from textures of impure dolomitic marbles from East Greenland. - J. Petrology, 23: 325343. Oxford

Bucher, K. - Frey, M. (1994): Metamorphism of Dolomites and Limestones. In: Petrogenesis of Metamorphic Rocks. - Springer Verl., Berlin-Heidelberg-New York, 171-189.

Burnham, C. W. (1959): Contact metamorphism of magnesian limestones at Crestmore, California. - Bull. geol. Soc. Amer., 70: 879-920.

Dubińska, E. (1997): Rodingites and amphibolites from the serpentinites surrounding Sowie Góry block (Lower Silesia, Poland): Record of supra-subduction zone magmatism and serpentinization. $-\mathrm{N}$. Jb. Mineral. Abh., 171: 239-279.

Dubińska, E. - Bylina, P. - Kozlowski, A. (2004): Garnets from Lover Silesia Rodingites: Constraints from their chemistry. - Mineralogical Soc. of Poland - Special papers, 24: 135-138.

Ehlers, K. - Hoinkes, G. (1987): Titanian Chondrodite and Clinohumite in Marbles from the Ötztal Crystalline Basement. - Mineral. Petrol., 36: $13-25$.

Foley, S. F. - Venturelli, G. - Green, D., H. - Toscani, L. (1987): The ultrapotassic rocks; characteristics, classification, and constraints for petrogenetic models. - Earth Sci. Rev. 24: 81-134.

Gaspar, J. C. (1992): Titanian clinohumite in the carbonatites of the Jacupiranga Complex, Brazil: Mineral chemistry and comparison with titanium clinohumite from other environments. - Amer. Mineral., 77: $168-178$.

Grew, E. S. - Yates, M. G. - Adams, P. M. - Kirkby, R. - Wiedenbeck, M. (1999): Harkerite and associated minerals in marble and skarn from Crestmore quarry, Riverside County, California and Cascade Slide, Adirondack Mountains, New York. - Canad. Mineral., 37: 277296.

Holub, F. V. (1997): Ultrapotassic plutonic rocks of the durbachite series in the Bohemian Massif: Petrology, geochemistry and petrogenetic intepretation. - Sbor. geol. Věd, 31: 5-26.

Holub, F. V. - Cocherie, A. - Rossi, P. (1997): Radiometric dating of granitic rocks from the Central Bohemian Plutonic Complex (Czech Republic): constraints on the chronology of thermal and tectonic events along the Moldanubian-Barrandian boundary. - C. R. Acad. Paris, Earth \& Planet. Sci., 325: 19-26.

Houzar, S. (2004): Metamorphosed carbonate rocks in the south-eastern margin of the Bohemian Massif. - Unpublished Thesis, Faculty of Science, Masaryk University, Brno, 154 p. (in Czech). 
Houzar, S. - Novák, M. (2001): Marbles in the south-eastern margin of the Bohemian Massif (Review). - Vlastivěd. Sbor. Vysočiny, Odd. Věd přír., 15: 3-33. (in Czech).

- (2006): Fluorine-rich clintonite in chondrodite marbles from the Moldanubian Zone, Czech republic. - Mineralogia Polonica, Special papers, 28: 92-94.

Kato, T. -Enami, M. - Zhai, M. (1997): Ultra-high-pressure (UHP) marble and eclogite in the Su-Lu UHP terrane, Eastern China. - J. Metamorph. Geol., 15: 169-182.

Kearns, L. E. - Kite, L. E. - Leavens, P. B. - Nelen, J. A. (1980): Fluorine distribution in the hydrous silicate minerals of the Franklin Marble, Orange County, New York. - Amer. Mineral., 65: 557-562.

Kerrick, D. M. (1991): Overview of Contact Metamorphism. In.: Kerrick, D. M. (Ed.) Contact Metamorphism. - Reviews in Mineralogy, 26: $1-12$.

Kotková, J. - Schaltegger, U. - Leichmann, J. (2003): 338-335 Ma old intrusions in the E Bohemian massif - a relic of the orogen-wide durbachitic magmatism in European Variscides. - J. Czech Geol. Soc., 48: 80-81.

Kretz, R. (1980): Occurrence, mineral chemistry and metamorphism of Precambrian carbonate rocks in a portion of the Grenville province. J. Petrology, 21: 573-620.

- (1983): Symbols for rock-forming minerals. - Amer. Mineral., 68: 277-279.

MacKinney, J. A. - Mora, C. I. - Bailey, S. W. (1988): Structure and crystal chemistry of clintonite. - Amer. Mineral., 73: 365-375.

Mottana, A. - Sassi, F. P. - Thompson, J. B. - Guggenheim, S. (2002): Micas: Crystal Chemistry and Metamorphic Petrology. - Reviews in Mineralogy, 46: $499 \mathrm{p}$.

Novák, M. (1988): Petrology of metamorphosed dolomitic rocks along northeastern border of the Moldanubicum. - Unpublished Thesis, Faculty of Science, Charles University, Praha. 127 p. (in Czech)

- (1989): Metamorfóza dolomitických hornin při severovýchodním okraji moldanubika. - Acta Mus. Moraviae, Sci. nat., 74: 7-51.

Novák, M. - Houzar, S. (1996): The HT/LP metamorphism of dolomite marbles in the eastern part of the Moldanubicum; a manifestation of heat flow related to the Třebíč durbachite Massif. - J. Czech geol. Soc., 41: 139-146.
Olesch, M. - Seifert, F. (1976): Stability and phase relations of trioctahedral calcium brittle micas (clintonite group). - J. Petrology, 17: 291-314.

Ottolini, L. - Cámara, F. - Bigi, S. (2000): An investigation of matrix effect in the analysis of fluorine in humite-group minerals by EMPA, SIMS, and SREF. - Amer. Mineral., 85: 89-102.

Powell, R. - Condliffe, D. M. - Condliffe, E. (1984): Calcite-dolomite geothermometry in the system $\mathrm{CaCO}_{3}-\mathrm{MgCO}_{2}-\mathrm{FeCO}_{3}$ : An experimental study. - J. Metamorph. Geol., 2: 33-41.

Rejl, L. - Sedlák, H. (1987): Contribution of geophysical mapping 1 : 25000 to the knowledge of geology and metallogenesis of the Třebíc and Jihlava Massifs. - Geol. Průzk. 29: 134-136. (in Czech).

Rice, J. M. (1979): Petrology of clintonite-bearing marbles in the Boulder aureole, Montana. - Amer. Mineral., 64: 519-526.

- (1980): Phase equilibria involving humite minerals in impure dolomitic limestones: Part II. Calculated stability of chondrodite and norbergite. - Contrib. Mineral. Petrology, 75: 205-223.

- (1983): Metamorphisn of rodingites: Part 1. Phase relations in a portion of the system $\mathrm{CaO}-\mathrm{MgO}-\mathrm{Al}_{2} \mathrm{O}_{3}-\mathrm{SiO}_{2}-\mathrm{CO}_{2}-\mathrm{H}_{2} \mathrm{O}$. - Am. J. Sci., 283A: 121-150.

Satish-Kumar, M. - Niimi, N. (1998): Fluorine-rich clinohumite from Ambasamudram marbles, Southern India: Mineralogical and preliminary FTIR spectroscopic characterization. - Miner. Mag., 62: 509-519.

Skippen, G. B. (1974): An experimental model for low pressure metamorphism of siliceous dolomitic marble. - Amer. J. Sci., 274: 487-509.

Shabynin, L. I. (1973): Formation of magnesian skarns. - Nauka, Moskva, 173 p., (in Russian).

Sugaki, A. - Campos, E. - Kojima, S. (2000): Mineralogy of the Panulcillo skarn copper deposit, Coquimbo Region, Chile. - Rev. geol. Chile, 27: $139-155$.

Ulmer, P. (1983): Monticellite-clintonite bearing assemblages at the southern border of the Adamello-Massif. - Redic. Soc. Ital. Mineral. e Petrol., 38: 617-628.

Woodford, D. T. - Sisson, V. B. - Leeman, W. P. (2001): Boron metasomatism of the Alta stock contact aureole, Utah: Evidence from borates, mineral chemistry, and geochemistry. - Amer. Mineral., 86: 513-533.

Zharikov, V. A. - Khodorevskaya, L. I. (1987): An experimental study of reactions of the clintonite origin. - Dokl. Akad. Nauk SSSR, Earth Science Sections, 294: 952-955 (in Russian).

\section{Asociace obsahující clintonit v chondroditových mramorech moldanubika z kontaktní aureoly třebíčského plutonu, Český masiv}

Clintonit, vzácná trioktaedrická slída s ideálním chemickým vzorcem Ca $\left(\mathrm{Mg}_{2} \mathrm{Al}_{3}\right)_{2}\left(\mathrm{SiAl}_{3}\right) \mathrm{O}_{10}(\mathrm{OH})_{2}$, byla zjištěna v chondroditových mramorech, uložených v migmatitech v blízkosti třebíčského plutonu (lokality Č́íhalín, Sokolí a Tasov). Clintonit v texturně rovnovážné asociaci s chondroditem, vzácněji se spinelem nebo chloritem I, náleží k mladší metamorfní asociaci těchto mramorů, která vznikla na úkor starší asociace Dol+Phl+Prg +Di(?) + Spl(?). Z akcesorických minerálů mramorů lze dále zmínit fluorapatit, pyrhotin, fluorit, zirkon a baddeleyit. Retrográdní stadium metamorfózy je reprezentováno vznikem hojného chloritu II na úkor clintonitu na př́mém kontaktu s chondroditem. Studovaný clintonit se vyznačuje vysokým poměrem Si/Al (0.52$0.60)$ a zejména vysokým obsahem $\mathrm{F}\left(\mathrm{X}_{\mathrm{F}}=0.11-0.26\right)$, čímž odpovídá fluorem nejbohatšímu dosud zjištěnému clintonitu. Asociující chondrodit (patrně je vzácně př́itomen i submikroskopický humit) je naopak fluorem relativně chudý $\left(\mathrm{X}_{\mathrm{F}}=0.45-0.67\right)$. Clintonit s chondroditem mohl vznikat zčásti $\mathrm{v}$ uzavřeném systému reakcemi, kdy zdrojem $\mathrm{F}$ byl flogopit a pargasit:

(5) $\mathrm{Dol}+\mathrm{Phl}+\mathrm{Di}+\mathrm{H}_{2} \mathrm{O}=\mathrm{Cli}+\mathrm{Chn}+\mathrm{Chl} \mathrm{I}+\mathrm{Cal}+\mathrm{CO}_{2}$ (Č́́halín)

(6) $\mathrm{Dol}+\mathrm{Prg}+\mathrm{H}_{2} \mathrm{O}=\mathrm{Cli}+\mathrm{Chn}+\mathrm{Cal}+\mathrm{CO}_{2}+\mathrm{Na}_{2} \mathrm{O} \pm \mathrm{K}_{2} \mathrm{O}$ (Sokolí, Tasov)

(7) $\mathrm{Dol}+\mathrm{Phl}+\mathrm{H}_{2} \mathrm{O}=\mathrm{Cli}+\mathrm{Chn}+\mathrm{Cal}+\mathrm{CO}_{2}+\mathrm{K}_{2} \mathrm{O}$ (Sokolí, Tasov)

Reálnější, a složením stabilních izotopů $\mathrm{C}$ a O v kalcitu chondroditových mramorů podpořený, je však vznik asociace clintonit+chondrodit za př́nosu $\mathrm{F}$, $\mathrm{H}_{2} \mathrm{O}$ a $\mathrm{SiO}_{2}$ z externího zdroje (zejména lokality Sokolí a Tasov) reakcí:

(8) $\mathrm{Dol}+\mathrm{Phl}$ (and/or Prg) $+\mathrm{SiO}_{2 \mathrm{aq}}+\mathrm{F}=\mathrm{Cli}+\mathrm{Chn}+\mathrm{Spl}+\mathrm{Cal}+\mathrm{CO}_{2}+\mathrm{K}_{2} \mathrm{O} \pm \mathrm{Na}_{2} \mathrm{O}$

Relativně vysoký podíl $\mathrm{F}$ ve fluidech patrně podporoval vznik clintonitu namísto spinelu a chloritu. Ve srovnání s dř́íve popsanými výskyty clintonitu v mramorech kontaktních aureol granitoidních plutonů, nevznikl studovaný clintonit na př́mém kontaktu magmatitů, ale v podmínkách periplutonické LP metamorfózy v aureole třebíčského plutonu, které lze přibližně odhadovat při předpokládaném $\mathrm{P}=200-400 \mathrm{MPa}$ na $\mathrm{T}=620-730{ }^{\circ} \mathrm{C}$ a $\mathrm{X}_{\mathrm{CO} 2}<0.6$. 